Original Research Paper

\title{
Comparative Collagen Distribution in the Small Intestine of Eidolon Helvum and Manis Tricuspis
}

\author{
Adefolaju Gbenga Anthony \\ Department of Pre-Clinical Sciences, Faculty of Health Sciences, University of Limpopo, Sovenga 0727, South Africa
}

\author{
Article history \\ Received: 13-09-2018 \\ Revised: 20-12-2018 \\ Accepted: 09-08-2019 \\ Email: gbenga.adefolaju@ul.ac.za
}

\begin{abstract}
The African tree pangolin has no teeth but consumes a highly tough and chitinous diet while the bat is frugivorous; this study was designed to compare the morphological adaptations of the small intestine of both mammals by investigating the collagen distribution and hydroxyproline/collagen content of their duodenum and ileum histologically and spectrophotometrically respectively. Ten animals each of both sexes were used. Portions of their small intestine were either fixed for routine histologic processing or homogenized and assayed for the hydroxyproline assay. Histological staining reveal that the collagenous distribution of duodenal submucosa and support of the villus core of the ileum is more prominent in the Pangolin than in the Bat. Data from the hydroxyproline colorimetric assay reveals that the Pangolin has significantly $(\mathrm{p}=0.00042)$ higher total collagen content than in the Bat. It is concluded that the Pangolin has evolved a higher expression and distribution of collagen in their small intestine as a mechanism of coping with their tough diets despite the lack of teeth.
\end{abstract}

Keywords: Bat, Pangolin, Collagen Stain, Hydroxyproline, Small Intestine

\section{Introduction}

The mammalian gastrointestinal tract comprise parts observed to exhibit morphological modifications due to their peculiar functions (Sherwood, 2008; Stevens and Hume, 2004) and as a result, different parts of the mammalian gut are commonly adapted to suit the dietary requirements of the different species. These variations are specialized to suit the dietary requirements of the particular species (Hildebrand and Goslow, 2001).

The work of Chivers and Hladic (1980) on the morphology of the gastrointestinal tract in primates and comparison with other mammals in relation to diet revealed that the ratio of stomach and large intestine to small intestine (by area, weight and volume) are low in frugivores and high in folivores; the continuous spread of coefficients reflect the different degrees of adaptation of these two dietary extremes.

The fruit Bat Eidolon helvum (Kerr, 1792) occupy a wide range of habitats, ranging from coastal forests to dry, arid regions (Adeniyi et al., 2012; Akobi et al., 2012; Hayman et al., 2012; Ossa et al., 2012; Nowak, 1999). The prime habitat however is tropical forests because of the abundance of food. African fruit-bats are found at elevations from sea level to $2000 \mathrm{~m}$. They typically roost in tall trees. They have also been found in caves (Adeniyi et al., 2012; Akobi et al., 2012; Hayman et al., 2012; Ossa et al., 2012; Nowak, 1999).
E. helvum weighs between $290 \mathrm{~g}$ and $230 \mathrm{~g}$ respectively. They are about $18 \mathrm{~cm}$ long. The wingspan of the African fruit bat is about $762 \mathrm{~mm}$ (Akobi et al., 2012; Ossa et al., 2012; Nowak, 1999). Manis tricuspis, the African tree pangolin, is one of eight extant Pangolins or scaly anteaters. Pangolins are unique looking mammals that are characterized by elongated bodies with long tails that are nearly completely covered by overlapping scales composed of agglutinated hair14 (Nowak, 1999; Ofusori et al., 2007). They have long claws on their forefeet for digging out ants and termites, their primary diet and a long, sticky, vermiform tongue, which has its muscular attachment to the pelvis (Nowak, 1999). Manis tricuspis is known to Africa only (Nowak, 1999; Ofusori et al., 2007). The pangolin skull is characterized by a lack of teeth, lack of zygomatic arches and an extremely reduced mandible (Schlitter, 2005). Each dentary has a single, dorsal, bony tooth-like protrusion. The tree Pangolin eats insects such as ants and termites from their nests. Pangolins use their 10-27-inch-long tongue which is coated with gummy mucus to funnel the insects into their mouth. The tongue is actually sheathed in the chest cavity all the way to the pelvic area (Schlitter, 2005).

Bat is frugivorous and Pangolin is insectivorous. Presumably, these mammals possess gut adaptations that allow them to efficiently process this mix of low and high digestibility component. It is to be anticipated that 
the Pangolin, which has no teeth unlike the Bat and some other mammals may possess such morphological and functional features such as a more sophisticated structural support adapted by their alimentary tracts to make them suitable for these high chitinous diet. Collagen is an important structural protein component of various connective tissues in animals (Di Lullo et al., 2002). The mucosa is composed of epithelium, a laminar propria (of collagen, elastic and reticular fibers) and muscular mucosae. The submucosa contains collagen fibers, fat and the submucosa nerve plexuses (Gray et al., 2005; Young et al., 2006). Ofusori et al. (2007) reported morphometric studies on the stomach of African Pangolins (Manis tricuspis), however no work has been done comparing the collagen support and hydroxyproline content of the small intestine of the Bat (E. helvum) and Pangolin (M. tricuspis), hence this work was designed to address this gap.

\section{Materials and Methods}

\section{Experimental Animals}

\section{Bats}

Ten (10) fruit Bats (Eidolon helvum) were captured with the assistance of experts (who possess State permits) at the Bats colony at Flower Gardens area of Government Reserved Area (GRA) of Ilorin, Kwara State, Nigeria. They were kept in bird cages prior to sacrifice.

\section{Pangolins}

Ten (10) Pangolins (Manis tricuspis) of both sexes were procured from the wild at Asejire area of Oyo State. Nigeria. They were brought to the Department of Anatomy, University of Ilorin Nigeria and kept overnight prior to sacrifice.

All animals were handled in conformity with the rules and guidelines of the animal rights committee of the University of Ilorin. They were evaluated and judged presumably healthy, fit enough to use for the study. Animals were anaesthetized, sacrificed and portions of the small intestines were fixed for histological processing and frozen for hydroxyproline analysis.

\section{Histological Analysis}

Tissues were fixed in $10 \%$ formol saline and processed for routine paraffin embedding. Serial sections were obtained at $5 \mu \mathrm{m}$ thickness from a rotary microtome and processed for the following staining procedures: Haematoxylin and Eosin (H\&E) and Masson Trichrome Stain, Gomori Method (Young et al., 2006). The Masson's trichrome stain is used to demonstrate supporting tissue elements, principally collagen (Young et al., 2006). As the name implies, the staining technique produces three colours: nuclei and other basophilic structures are stained blue; collagen is stained blue or green depending on which variant of the technique is used; and cytoplasm, muscle, erythrocytes and keratin are stained bright red as demonstrated by Young et al. (2006). The Olympus microscope BX51 and digital camera DP70 (Olympus, Tokyo, Japan) was used for imaging purposes.

\section{Spectrophotometric Analysis of Hydroxyproline as an Indication of Collagen levels}

Hydroxyproline is a major constituent of collagen and contains about $12.5-13.5 \%$ of its amino acid composition. As a result of its restricted distribution in collagen, the hydroxyproline content accurately reflects the amount of collagen in biological samples. Quantifying hydroxyproline has been utilized for evaluating tissue collagen deposition. Assay procedure was followed as described by Reddy and Enwemeka (1996). Briefly, tissue homogenates and hydroxyproline standards (Sigma, St Louis MO, USA) were hydrolyzed in $2 \mathrm{~N}$ sodium hydroxide (Sigma, St Louis MO, USA) by incubating at $120^{\circ} \mathrm{C}$ for $20 \mathrm{~min}$. The hydrolyzed samples were then mixed with a buffered Chloramine-T reagent (Sigma, St Louis MO, USA) and the oxidation was allowed to proceed for $25 \mathrm{~min}$ at room temperature. Coloration was then developed with the addition of Ehrlich's reagent (Sigma, St Louis MO, USA) and the optical density of the reddish purple complex was measured at $550 \mathrm{~nm}$ using a microplate reader (Anthos 2010 Model 17-550 Austria). In Fig. 1, absorbance values were plotted against the concentration of standard hydroxyproline and the presence of hydroxyproline in unknown tissue extracts was determined from the standard curve (Hoerstrup et al., 1999; Kliment et al., 2011; Reddy and Enwemeka, 1996).

\section{Statistical Analysis}

Statistical analysis was performed using JMP® (Version 10.0 SAS Institute Inc., Cary, NC). Data are reported as mean + SEM. After verifying the normal distribution and the homogeneity of the variance using an $F$ test $(\mathrm{p}<0.05)$, a one-way analysis of variance (where a significance level of $\mathrm{p}<0.05$ was set) was used to compare the results.

\section{Results}

\section{Histological Analysis}

A comparative demonstration of the duodenal mucosa of the Bat is thrown into folds Plicae Circulares (PCS) unlike in the Pangolin (Fig. 2A and 2B). Thicker submucosal Brunner's glands are observed in the Pangolin compared to the Bats. The micrographs shown in Fig. 3 demonstrate the diffuse distribution of Collagen (green) in the duodenum of the Bat and the Pangolin. 


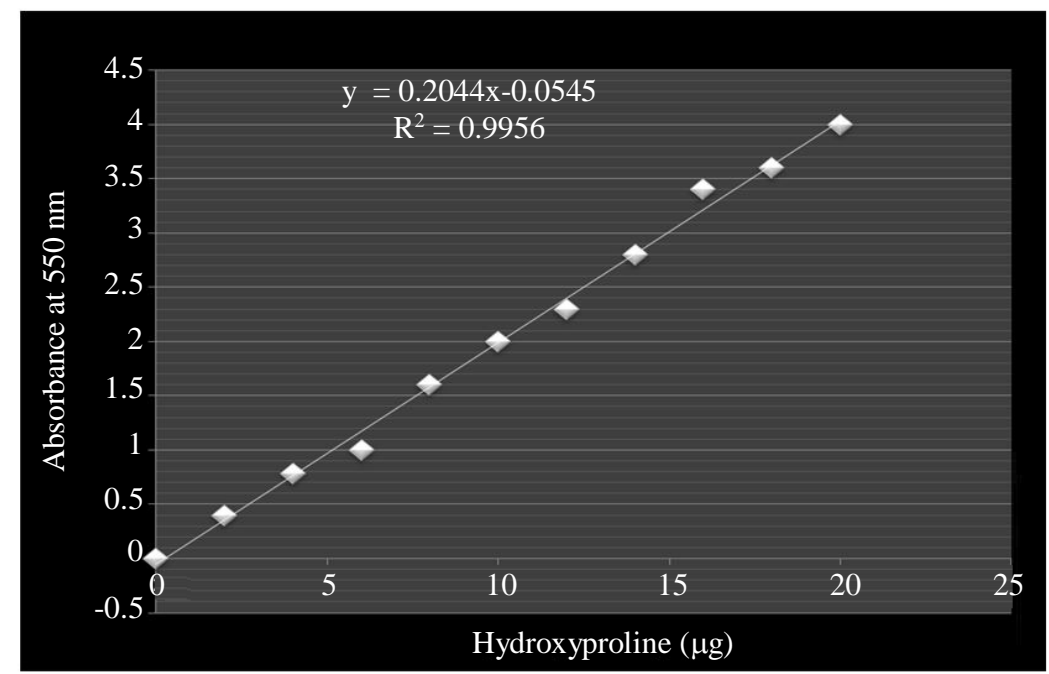

Fig. 1: Standard curve for hydroxyproline assay. Serial dilutions $(2-20 \mu \mathrm{g})$ of standard hydroxyproline was prepared from stock solution. OD values of the standards are plotted on the $y$-axis and the Hydroxyproline standard concentrations are plotted on the $\mathrm{x}$-axis
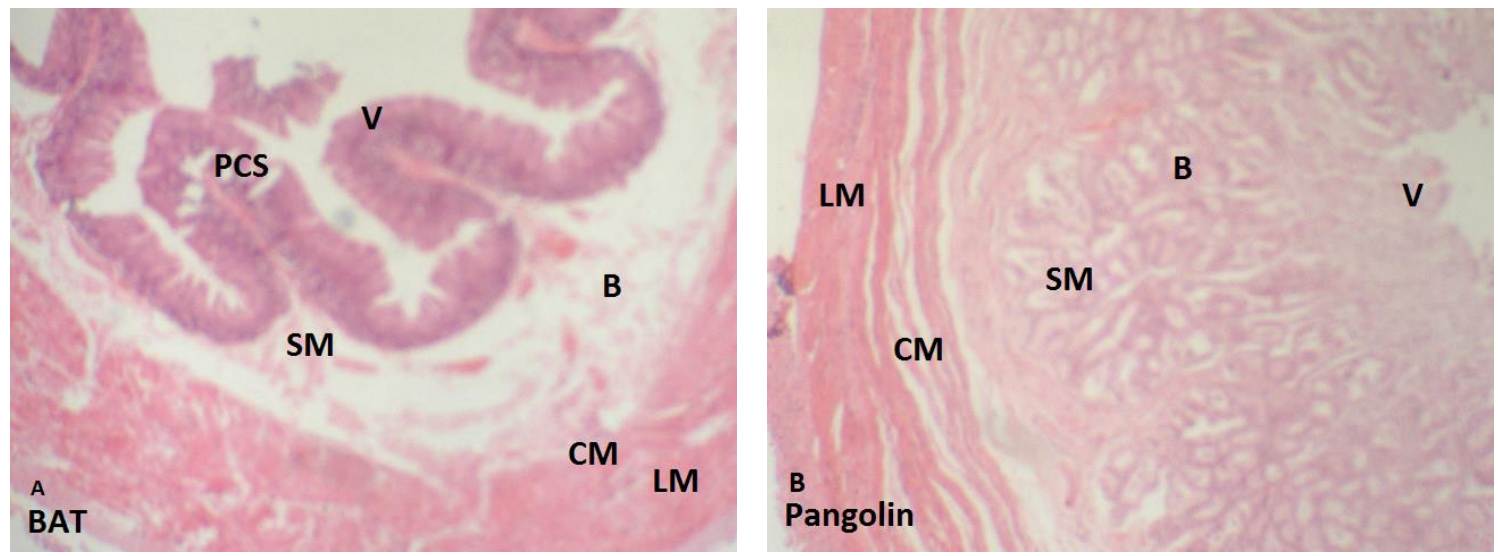

Fig. 2: Photomicrograph of the duodenum of the Bat (A) and Pangolin (B). H\&E X100. V (villi), LM (Longitudinal muscle), CM (Circular Muscle), B (Brunner's gland) SM (Submucosa)
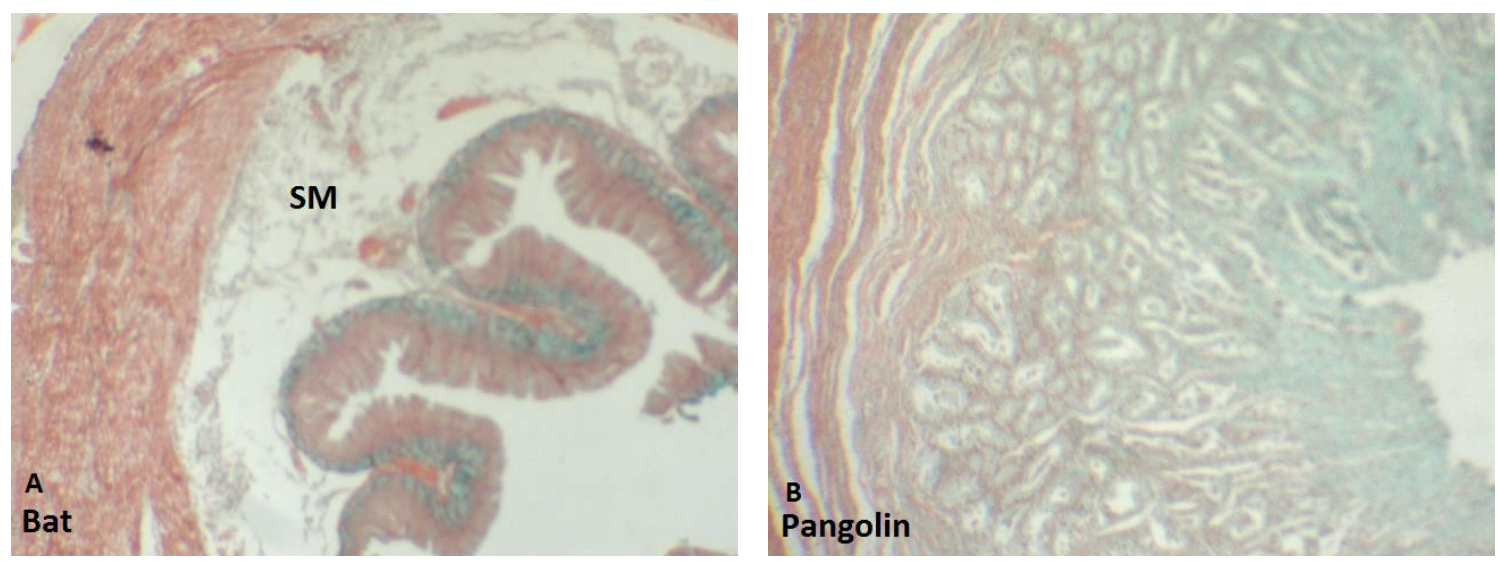

Fig. 3: Photomicrograph of the duodenum of the Bat (A) and Pangolin (B). Masson's Trichrome X100 Micrographs show diffuse distribution of Collagen (green). Collagenous support of the villus is more widespread and prominent in the Pangolin than in the Bat 

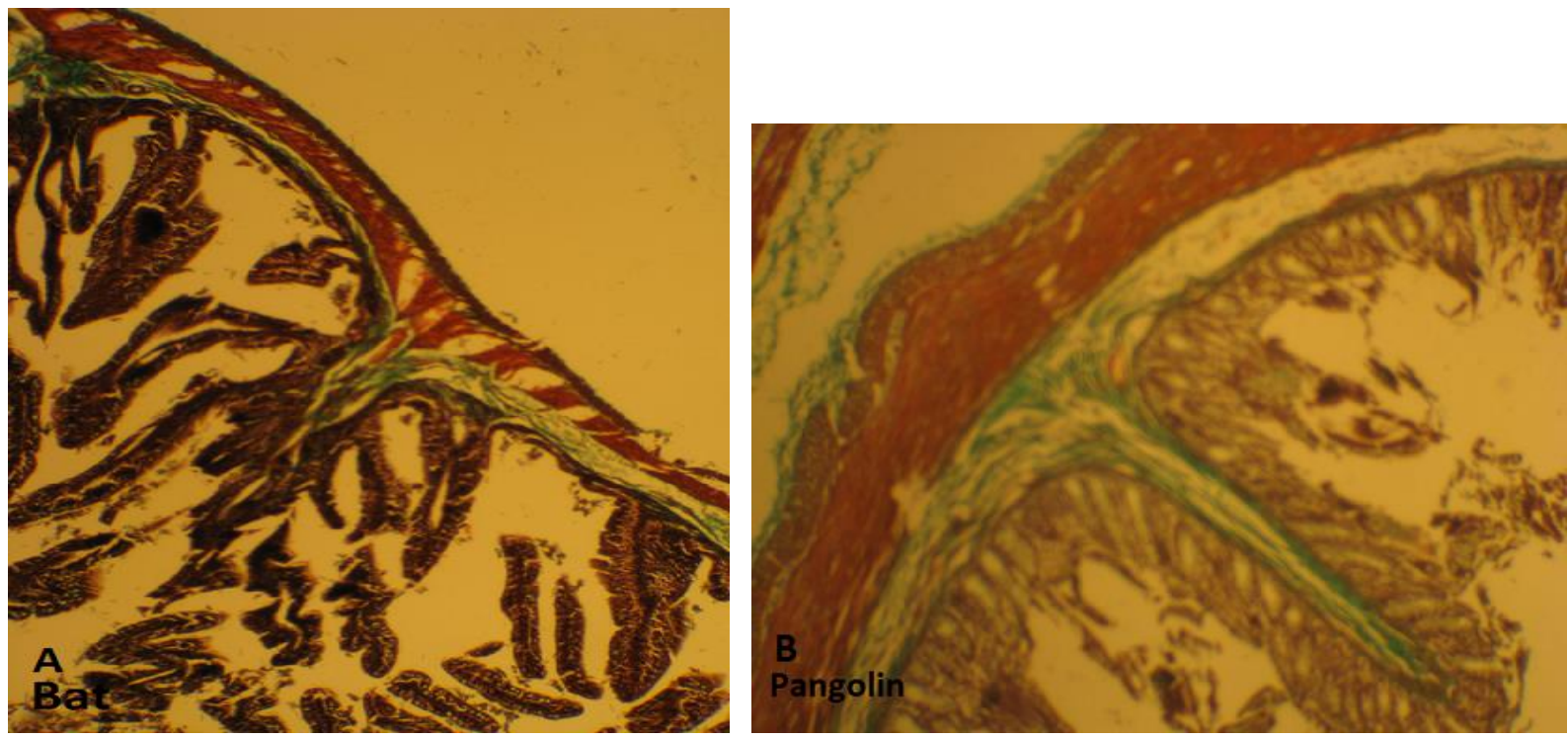

Fig. 4: Photomicrograph of the ileum of Bat (A) and Pangolin (B). Masson's Trichrome X100 Micrograph show diffuse distribution of Collagen. Collagen (green) provides support for the villus core. It is more prominent in the Pangolin than in the Bat

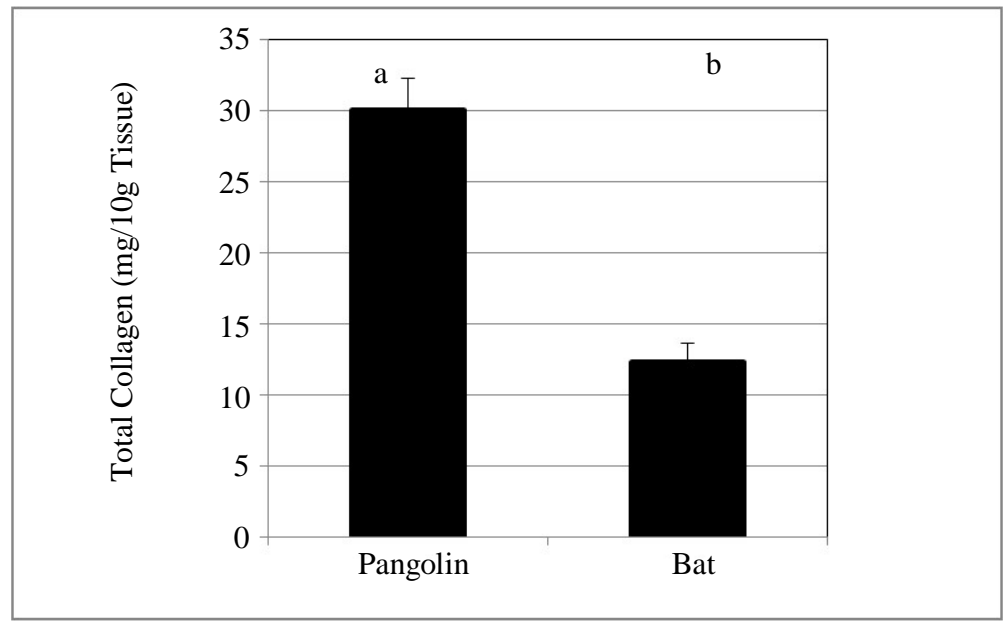

Fig. 5: Comparison of total collagen content of the small intestine of the Pangolin (M. Tricuspis) and Bat (E. helvum) Data (mean \pm $\mathrm{SEM})$ are Total Collagen (mg/10g tissue), representative of 3 independent experiments. Groups not connected by the same letter are significantly $(\mathrm{p}=0.00042)$ different

Collagenous support of the villus is more prominent in the Pangolin than in the Bat. Figure 4 demonstrate the localization of Collagen (green) in the villus core of the ileum of the Bat and the Pangolin. The collagenous support for the villus core of the ileum is more prominent in the Pangolin than in the Bat.

\section{Analysis of Total Collagen by Hydroxyproline Colorimetric Assay}

The hydroxyproline level of each sample is converted into the total collagen level by the following equation: Hydroxyproline level $(\mu \mathrm{g} / \mathrm{mg}) \times 100 / 13.5=$ Collagen level $(\mu \mathrm{g} / \mathrm{mg})$.
Hydroxyproline content of collagen amino acid composition is considered to be $13.5 \%$ (Kliment et al., 2011). Figure 5 shows that in $100 \mathrm{~g}$ of small intestinal tissue obtained from both groups of mammals, the Pangolin has significantly higher total collagen content than in the Bat. The amount of collagen in $100 \mathrm{mg}$ of Pangolin small intestinal tissue is more than double the amount present in Bat tissue.

\section{Discussion}

This study sought to investigate the morphological adaptations of the small intestine of a toothless mammalian specie - (Manis tricuspis) that consumes a 
highly chitinous arthropod-exoskeleton diet and compare with the morphology of the another mammalian specie (Eidolon helvum) that consumes relatively soft frugivorous diet. Since Pangolins lacked teeth, it was to be expected that there would be histological adaptions of its gut to manage the demands of processing the tough protective components of arthropod exoskeletons.

The results of this study show that there is a more diffuse distribution of collagen and collagenous villous support in the duodenum and ileum of the Pangolin than in the Bat as demonstrated by the Mallory's trichrome stain. This observation is further corroborated by the spectrophotometric analysis of total collagen content in the small intestines of both mammalian species. Results from this study show that the total collagen content in $10 \mathrm{~g}$ of small intestinal tissue of Pangolins is more than double that of the Bats. The results also show that submucosal Brunner's glands are thicker in the Pangolin compared to the Bats. This structural modification may be related the need for more mucus production in the Pangolin because of their highly chitinous diet, a result of which mucosa surfaces will require more lubrication. The presence of collagen permits the considerable changes in transverse and longitudinal dimensions that accompany peristalsis (Gray et al., 2005), whilst still providing adequate support and strength which is required more in the pangolin because of its diet. The morphological organisation of the small intestine in both animals conforms to that of a typical tubular organ as described previously (Coico et al., 2003; Hildebrand and Goslow, 2001; Young et al., 2006). The findings of this study disagree with the observations of Ofusori et al. (2008) regarding the absence of collagen fibres in the submucosa of bat ileum. The reason for this might be that this study utilised a more appropriate Masson's trichrome stain for the demonstration of collagen. Enzymatic activity levels that highlight the functional adaptations have been previously reported. For instance, alkaline and acid phosphatase activities were found to be significantly higher in the bat's small intestine compared to the pangolin (Adefolaju et al., 2009). The higher ALP activities in the bat has been linked with the faster transit times in the bat gut as alkaline phosphatase facilitates transport across intestinal mucosa (Lynes et al., 2011; Nassir et al., 2007).

\section{Conclusion}

The findings of this study provide further information on the morphological adaptations and mechanisms evolved by the Pangolin to cope with its unique diet. The Pangolin has developed a higher expression and distribution of collagen in their small intestine as a mechanism of coping with their tough diets despite the lack of teeth.

\section{Acknowledgement}

The author thanks Mr BA Falana of the Department of Anatomy, College of Helath Sciences, University of Osun, Nigeria for assisting with this work.

\section{Ethics}

This article is original and contains unpublished material. The corresponding author declares that there are no ethical issues involved.

\section{References}

Adefolaju, G.A., A.E. Caxton-Martins, B.U. Enaibe, O.T. Alabi and M.S. Ajao, 2009. Acid and Alkaline Phosphatase activities in the small intestine of the Rat (Rattus norvegicus), Bat (Eidolon helvum) and Pangolin (Manis tricuspis). Intern. J. Pharm., 6: 9351.

Adeniyi, T., A. Tijani, D. Adekomi and T. Abayomi, 2012. A comparative study of the lateral geniculate body of rat (Rattus norvegicus), bat (Eidolon helvum) and pangolin (Manis tricuspis). Glob J. Health Sci., 4: 118-125.

Akobi, B., O. Aboderin, T. Sasaki and A. Shittu, 2012. Characterization of Staphylococcus aureus isolates from faecal samples of the Straw-Coloured Fruit Bat (Eidolon helvum) in Obafemi Awolowo University (OAU), Nigeria. BMC Microbiol., 12: 279.

Chivers, D.J. and C.M. Hladik, 1980. Morphology of the gastrointestinal tract in primates: Comparisons with other mammals in relation to diet. J. Morphol., 166: 337-386.

Coico, D.J., G. Sunshine and E. Benjamini, 2003. Immunology: A short course. John Wiley and Sons, Hoboken NJ.

Di Lullo, G.A., S.M. Sweeney, J. Korkko, L. Ala-Kokko and J.D. San Antonio, 2002. Mapping the ligandbinding sites and disease-associated mutations on the most abundant protein in the human, type I collagen. J. Biol. Chem., 277: 4223-4231.

Gray, H., S. Standring, H. Ellis and B.K.B. Berkovitz, 2005. Gray's anatomy: The anatomical basis of clinical practice. Elsevier Churchill Livingstone, Edinburgh; New York.

Hayman, D.T.S., R. McCrea, O. Restif, R. Suu-Ire and A.R. Fooks et al., 2012. Demography of straw-colored fruit bats in Ghana. J. Mammal., 93: 1393-1404.

Hildebrand, M. and G. Goslow, 2001. Analysis of vertebrate structure. Wiley, New York.

Hoerstrup, S.P., G. Zünd, Q. Ye, A. Schoeberlein and A.C. Schmid et al., 1999. Tissue engineering of a bioprosthetic heart valve: Stimulation of extracellular matrix assessed by hydroxyproline assay. ASAIO J., 45: 397-402. 
Kerr, R., 1792. The animal kingdom, or zoological system of the celebrated Sir Charles Linnaeus. Class 1. Mammalia. John Murray, London.

Kliment, C.R., J.M. Englert, L.P. Crum and T.D. Oury, 2011. A novel method for accurate collagen and biochemical assessment of pulmonary tissue utilizing one animal. Int. J. Clin. Exp. Pathol., 4: 349-355.

Lynes, M., S. Narisawa, J.L. Millan and E.P. Widmaier, 2011. Interactions between CD36 and global intestinal alkaline phosphatase in mouse small intestine and effects of high-fat diet. AJP: Regulatory, Integrative Comparative Physiol., 301: R1738-R1747.

Nassir, F., B. Wilson, X. Han, R.W. Gross and N.A. Abumrad, 2007. CD36 is important for fatty acid and cholesterol uptake by the proximal but not distal intestine. J. Biol. Chem., 282: 19493-19501.

Nowak, R.M., 1999. Walker's mammals of the world. JHU Press.

Ofusori, D.A., A.E. Caxton-Martins, A.O. Komolafe, K.A. Oluyemi and A.O. Adeeyo et al., 2008. A comparative study of the ileum in rat (Rattus norvegicus), bat (Eidolon helvum) and pangolin (Manis tricuspis) as investigated using histological method. Int. J. Morph., 26: 137.
Ofusori, D.A., A.E. Caxton-Martins, T.K. Adenowo, G.B. Ojo and B.A. Falana et al., 2007. Morphometric study of the stomach of African pangolin (Manis tricuspis). Sci. Res. Essays, 2: 465-467.

Ossa, G., S. Kramer-Schadt, A.J. Peel, A.K. Scharf and C.C. Voigt, 2012. The movement ecology of the straw-colored fruit bat, Eidolon helvum, in subSaharan Africa assessed by stable isotope ratios. PLoS ONE, 7: e45729-e45729.

Reddy, G.K. and C.S. Enwemeka, 1996. A simplified method for the analysis of hydroxyproline in biological tissues. Clin. Biochem., 29: 225-229.

Schlitter, D.A., 2005. Order Pholidota. In: Mammal Species of the World, A Taxonimic and Geographic Reference, Wilson, D.E. and D.M. Reeder (Eds.), John Hopkins University Press, Baltimore, pp: 530-531.

Sherwood, L., 2008. Human physiology: From cells to systems. Cengage Learning.

Stevens, C.E. and I.D. Hume, 2004. Comparative physiology of the vertebrate digestive system. Cambridge University Press.

Young, B., J.S. Lowe, A. Stevens, J.W. Heath and P.J. Deakin, 2006. Wheater's functional histology. Elsevier Health Sciences. 